\title{
DEVELANDO LA ACCIÓN PEDAGÓGICA EN UN SALÓN DE CLASE DE EDUCACIÓN INICIAL
}

\section{Ana Lupita Chaves Salas}

RESUMEN: El artículo resume el proceso investigativo y los hallazgos de un estudio que se llevó a cabo en una aula de educación inicial. La investigación responde a la necesidad de interpretar la realidad educativa en el contexto del aula con el propósito de promover la reflexión crítica sobre la teoría y la práctica pedagógica que asumimos y llevamos a cabo las y los educadores del nivel inicial, tomar conciencia de lo que hacemos y promover procesos de transformación de la práctica educativa.

El interés específico se dirigió a interpretar las relaciones de poder que se generan en la interacción del aula y analizar los procesos que siguió una educadora para incentivar la apropiación de la lengua escrita a partir de la filosofía de Lenguaje Integral.

Palabras clave: EDUCACION INICIAL / EDUCACION PREESCOLAR/ AULAS/ RELACIONES DE PODER/ INTERACCION EN EL AULA/ LENGUAJE INTEGRAL/ PRACTICA PEDAGÓGICA/ TEORIAS/

\section{INTRODUCCIÓN}

En el presente artículo se presenta el proceso investigativo que llevé a cabo en un salón de clase de educación inicial que seguía los principios de la filosofía del Lenguaje Integral.

El estudio lo realicé durante el ciclo lectivo de 1999, y se efectuó en un centro infantil público que atendía niñas y niños desde los cinco años y tres meses hasta siete años de edad.

El propósito de la investigación fue observar e interpretar las interacciones que se generaban en el contexto de un aula: las actitudes de la educadora, las interacciones de las niñas y los niños, las relaciones de poder, las prácticas y lenguaje sexista y la organización de los procesos iniciales de lecto-escritura. Quise analizar diversos factores que intervienen en la realidad compleja y singular del aula; pues como bien lo indican José Gimeno y Ángel Pérez (1993), solamente analizando y reflexionando sobre esta realidad se puede entender y transformar la práctica pedagógica.

\footnotetext{
${ }^{1}$ Doctora en Educación con énfasis en Investigación Educativa de la Universidad Estatal a Distancia en Costa Rica. Maestría en Educación de Adultos de la Universidad de Costa Rica. Bachiller y Licenciada en Educación Preescolar de la Universidad de Costa Rica. Directora del Instituto de Investigación para el Mejoramiento de la Educación Costarricense (IIMEC). Profesora de la Escuela de Formación Docente. Sección de Preescolar.

e-mail: jorgeurena@amnet.co.cl
} 
En el primer apartado presento las motivaciones personales que me llevaron a investigar sobre este tema, y hago una descripción de los valores y teorías que asumí para el abordaje del problema bajo estudio. Posteriormente, desarrollo el proceso metodológico, enmarcado dentro del paradigma cualitativo, el cual me permitió observar, comprender e interpretar las interacciones que se producen en la cotidianidad del aula.

Finalmente presento los hallazgos más relevantes de la investigación y las reflexiones finales en torno a los procesos de transformación que requiere nuestra práctica pedagógica, para así contribuir a construir una sociedad más solidaria y democrática.

\section{CONSTRUYENDO UTOPÍAS EN EL CONTEXTO ESCOLAR}

La escuela como institución social reproductora de desigualdades y transmisora y constructora de valores, debe replantear su función y contribuir, junto con otras instituciones de la sociedad, a la formación de las conciencias, a la elaboración del conocimiento y a su distribución igualitaria mediante prácticas equitativas y democráticas (Freire, Paulo,1997).

Desde esta perspectiva, mi interés se dirigió a promover la reflexión crítica sobre la teoría y la práctica pedagógica que asumimos y llevamos a cabo las educadoras y los educadores del nivel inicial. Para ello consideré importante analizar el currículo como proyecto político-ideológico, cultural y pedagógico, en cuanto a: su función social, fundamentos teóricos, papel de las personas que intervienen en su aplicación, relaciones de poder, uso de lenguaje, estereotipos de género, estrategias de enseñanza y de evaluación en torno a los procesos iniciales de lectoescritura y pertinencia del significado de los contenidos; ya que como lo define Alicia de Alba, el curriculum es una:

Propuesta político-educativa conformada por la síntesis de elementos culturales (conocimientos, valores, costumbres, creencias, hábitos) que conforman una propuesta político educativa pensada e impulsada por diversos grupos y sectores sociales cuyos intereses son diversos y contradictorios, aunque algunos tienden a ser dominantes y hegemónicos, y otros tienden a oponerse y resistirse a tal dominación o hegemonía. Síntesis a la cual se arriba a través de diversos mecanismos de negociación e imposición social (1994, p. 90)

De ahí que, el curriculum como proyecto político, responde a un contexto sociohistórico específico, donde intervienen diversos grupos sociales, por lo que una de sus características 
es su no neutralidad. Por ello, es necesario cuestionar la visión de mundo-sociedad y ser humano que orienta la propuesta curricular que desarrollamos en el aula, ya que esta va a determinar la forma como concebimos la relación entre quien conoce y lo que se va a conocer, es decir, la manera en que el ser humano se apropia del conocimiento, y dependiendo de esta concepción, así vamos a organizar el proceso de enseñanza y aprendizaje. Al respecto, Alicia Gurdián indica que el curriculum "se construye a partir de tres niveles interdependientes: El nivel ontológico-axiológico determina o define el siguiente nivel, esto es el epistemológico, el cual a su vez define o determina el metodológico. Es más, cualquier cambio en uno de estos niveles afecta a los otros" (2001, p. 19).

En este contexto, es importante develar la acción pedagógica en un aula del nivel inicial, pues esta práctica responde al proyecto del centro educativo, y este, a su vez, al Sistema Educativo Nacional. Quise descubrir los significados que se transmiten mediante las relaciones de poder que se dan en el salón de clase y cuestionar las prácticas de aprestamiento que se promueven en los centros infantiles y que responden a métodos tradicionales de enseñanza con el propósito de "preparar" a las niñas y a los niños para su ingreso al primer grado de la Educación General Básica.

El aprestamiento promueve la repetición de ejercicios para el desarrollo de destrezas y de habilidades por parte de los niños y las niñas, y responde a un interés técnico de la enseñanza orientado al control del aprendizaje del estudiante. En este proceso se concibe al estudiante como un agente pasivo que aprende a través de la experimentación, y no como un sujeto que construye y reconstruye el conocimiento en interacción con su medio sociocultural. Por ello, considero necesario, que las educadoras y los educadores reflexionemos sobre nuestra práctica pedagógica, los principios que la sustentan, los significados que transmitimos, el sexismo lingüístico y la subjetividades que ayudamos a construir, puesto que sólo así podremos explicar, replantear y mejorar nuestra acción educativa como práctica liberadora que lleve a un verdadero desarrollo humano.

Así las cosas, mi interés se orientó a develar la acción pedagógica que se desarrollaba en un salón de clase de educación inicial que pone en práctica la filosofía del Lenguaje Integral, la cual promueve prácticas pedagógicas diferentes a las tradicionales con el propósito de que los niños y las niñas se apropien de la lengua escrita, donde aprender el lenguaje es aprender a dar significado, aprender a darle sentido al mundo; donde las experiencias, las formas de hablar y los conocimientos de las estudiantes y los estudiantes se constituyen en un excelente recurso para construir nuevos conocimientos a partir de 
acciones contextualizadas y significativas que prepara e personal docente. En este contexto se respeta al estudiante, su origen y su forma de hablar (Arellano, 1995).

El Lenguaje Integral no es un método para enseñar a leer y a escribir sino es una nueva concepción de lenguaje y de ser humano en interacción, donde educadores y educadoras y estudiantes comparten el poder y tienen un papel protagónico en el proceso de enseñanza y de aprendizaje como investigadores de contextos socioculturales para conocer diferentes realidades (Goodman, K, 1970 y Goodman y Goodman,1993).

Por esta razón, consideré importante observar e interpretar la acción pedagógica que se fundamenta en la filosofía del Lenguaje Integral en el nivel de educación inicial de nuestro país, con el propósito de iniciar la discusión colectiva en torno a los procesos iniciales de lectoescritura y a la construcción de las subjetividades.

Lo anteriormente expuesto motivó a indagar sobre la siguiente interrogante generadora:

¿Cómo se construye la acción pedagógica a partir de la filosofía del Lenguaje Integral en un salón de clase del nivel de educación inicial del ciclo de transición?

De éste se desprenden otras preguntas, tales como:

1. ¿Cuál es el papel que desempeña la educadora en su práctica pedagógica?

2. ¿Cómo se dan los procesos de socialización en el aula mediante la selección de contenidos, la participación, la organización del espacio y del tiempo?

3. ¿Cómo se reproducen estereotipos de género en el salón de clase ?

4. ¿De qué manera la educadora orienta los procesos iniciales de lectoescritura en los niños y las niñas?

Para dar respuesta a las preguntas asumí el paradigma cualitativo con el propósito de estudiar la realidad-realidades en el contexto cultural del aula.

\section{EL CAMINO PARA INTERPRETAR LA COTIDIANIDAD DEL AULA}

Desde el paradigma cualitativo me acerqué a la situación bajo estudio. Realicé la investigación interactuando con una educadora y un grupo de catorce niños y dieciocho niñas de cinco años y tres meses a siete años de edad, de un jardín infantil público de Moravia que atiende una población de nivel socioeconómico bajo y medio-bajo. 
La estrategia de investigación fue inductiva y progresiva, me incliné por el método hermenéutico-dialéctico para comprender e interpretar los significados que se transmitieron en las interacciones del salón de clase (Martínez, Miguel,1989).

El proceso investigativo se desarrollo en las siguientes etapas:

I. Reflexiones individuales

II. Elaboración teórica

III. Reflexiones colectivas

IV. Identificación del tema

V. Negociación de entrada

VI. Inmersión al salón de clase

VII. Compartiendo la información

VIII. Análisis de datos

Estas etapas no se dieron de manera lineal, sino que la mayoría de ellas estuvieron presentes durante todo el proceso investigativo.

El proceso que viví en cada una de esas etapas lo describo a continuación:

\section{Etapa. Reflexiones individuales}

Por mi experiencia y reflexión en el campo de la educación inicial, la investigación se concentró en las prácticas de aprestamiento que se promueven en los centros de educación infantil para "preparar" a los niños y a las niñas para aprender a leer y a escribir. Esas prácticas me preocupaban pues respondían a concepciones empiristas y conductistas de la educación que no contribuyen a la formación de personalidades creativas ni críticas. Por otra parte, las niñas y los niños rápidamente se aburren de esas prácticas sin significado ni sentido para ellas y ellos. De ahí que, quise realizar el estudio de una práctica pedagógica, que consideraba diferente, en cuanto al abordaje de los procesos iniciales de lectoescritura.

Posteriormente, observé las relaciones de poder que se generaban en el aula y su similitud con la prácticas sociales de la cultura capitalista-patriarcal. Empecé a percibir las desigualdades que conciente e inconcientemente se producen en el contexto escolar y me preocupé por pensar en estrategias que promovieran cambios en esas interacciones.

La reflexión individual estuvo presente durante todo el proceso de investigación: reflexionaba sobre lo leído lo observado y lo que iba descubriendo en la interacción con las 
personas participantes. Fue un ir y venir, un pensar y un repensar, un descubrir y un redescubrir lo me ayudó a comprender e interpretar la realidad del aula que observaba y a la vez, mi práctica pedagógica como profesora universitaria.

\section{Etapa. Elaboración teórica}

Para iniciar el estudio, partí de premisas teóricas en torno al problema a investigar fundamentadas en autores como: Lev Vigotsky (1972), Paulo Freire(1997), Emilia Ferreiro (1983), Yetta y Kenneth Goodman $(1989,1993)$ y M. Halliday (1986).

Posteriormente, con la lectura y relectura de autores como: Jürgen Habermmas (1984), Alicia Gurdián (1997,1998), Jennifer Gore (1996), Henry Giroux (1997 y 1997a), Peter McLaren (1997), entre otros, hice otro acercamiento teórico con una visión más sociopolítica de la educación que psicologista, este nuevo acercamiento me permitió analizar la realidadrealidades del aula de una manera diferente e incluí como tema de análisis las relaciones de poder que se generan en el contexto escolar.

La elaboración teórica no fue solamente una segunda etapa de la investigación sino que estuvo presente, siempre, desde el primer momento en que se inició el estudio.

\section{Reflexiones colectivas}

Las reflexiones con las compañeras de trabajo en la Sección de Educación Preescolar de la Universidad de Costa Rica, y con la educadora con la que realicé la investigación sobre cómo orientábamos los procesos iniciales de lecto-escritura en las niñas y los niños menores de siete años, se venían dando desde hacía mucho tiempo. Las lecturas, el asistir a conferencias, a seminarios y a congresos, nos hacen reflexionar sobre la necesidad de cambiar, de buscar alternativas pedagógicas diferentes en torno a éste tema. Ésta fue una de las razones primordiales para realizar la investigación.

Una vez iniciado el estudio, las reflexiones y el diálogo constante con la educadora y con una compañera de estudio que realizaba una investigación en un aula de primer grado, fue una de las experiencias más valiosas de la investigación, pues se confrontaban puntos de vista, se compartieron vivencias y se buscaban explicaciones a lo que acontecía en el aula. Realmente fue una experiencia de aprendizaje colectivo. En éstos diálogos, se toma conciencia de que el currículo, como proyecto político-ideológico, cultural y pedagógico no es neutro, lo que me permitió observar de manera diferente lo que sucedía en el salón de clase. 


\section{Identificación del tema}

Los temas bajo estudio emergieron de mis vivencias como estudiante de educación inicial, como educadora de niños y niñas menores de siete años y como profesora universitaria.

Los procesos iniciales de lecto-escritura es un tema interesante por las implicaciones que trae, como herramienta para que los seres humanos conozcan el mundo y se desarrollen en él. Igualmente, el tema de las relaciones de poder en el aula, me apasionó puesto que muchas veces, como educadoras, pensamos que estamos promoviendo acciones que promueven la justicia y la democracia, pero de manera ingenua e irreflexiva, con nuestra práctica cotidiana, estamos reproduciendo las desigualdades sociales de la cultura hegemónica.

\section{Etapa. Negociación de entrada}

La investigación quería realizarla en el grupo de la educadora cuya práctica pedagógica conocía, por tal razón dos meses antes de ingresar a la aula negocié, formalmente la entrada a la institución, para ello expuse el proyecto de investigación a la directora y a la educadora, con el fin de interesarlas y lograr su confianza (Woods, Peter,1993), el proceso que seguí lo realicé de la siguiente manera:

- Me puse en contacté con la educadora con la que deseaba realizar la investigación, le expuse en términos generales, los propósitos del estudio y el deseo de hacer la labor de campo con su grupo de niñas y niños, a lo cual accedió con gusto. Es importante resaltar que desde hacía aproximadamente dos años, desde 1996, habíamos venido conversando sobre la necesidad de hacer un estudio de este tipo.

- $\quad$ En el mes de diciembre de 1998, visité el centro educativo, conversé con la directora, traté asuntos como: la importancia del estudio, el papel de la investigadora, interés por interpretar la realidad-realidades y necesidad de contar con su apoyo. Le entregué una carta solicitando la autorización y un resumen del proyecto. La directora aceptó amablemente

- El propósito de negociar la entrada fue conseguir el ingreso a la institución donde se realizaría el trabajo de campo. 
- $\quad$ Con el fin de que los niñas y las niñas me percibieran como parte de su grupo inicié las observaciones el primer día de clases en el mes de febrero de 1999.

\section{Etapa. Inmersión al salón de clase}

Con una gran expectativa ingresé al aula. Desde el primer momento, hubo aceptación por parte del grupo de niños y niñas y por la educadora. Me ubiqué en una silla pequeña y observé las interacciones, llevaba mi diario de campo y un lapicero pues había decidido anotar todo lo que acontecía; sin embargo, ese primer día hice observaciones generales, no muy detalladas para que los niños y las niñas no me percibieran como una persona que escribía y escribía, sino como una más del grupo.

Las observaciones las realicé durante el ciclo lectivo de 1999 de la siguiente manera:

- $\quad$ Dos veces por semana en los meses de febrero, marzo y abril.

- Una o dos veces por semana durante los meses de junio, julio y agosto

- $\quad$ En agosto ingresó al salón de clase una estudiante que realizó su práctica profesional durante el segundo semestre, por tal situación, observé las interacciones del aula únicamente cuando la educadora era la que dirigía el trabajo con los niños y las niñas.

- En setiembre y octubre no visité la institución.

- $\quad$ Una vez por quincena durante los meses de noviembre y diciembre.

Las observaciones las realicé en períodos de dos horas, aproximadamente, y seleccioné los momentos de la jornada diaria más relevantes para el propósito del estudio: actividades iniciales, conversación, juego en áreas, educación física y merienda. En este proceso, las grabaciones, los vídeos y la fotografía, se constituyeron en un excelente apoyo para representar la realidad-realidades del aula.

Las notas que tomaba en mi diario de campo las transcribía generalmente, el mismo día que realizaba la observación, sin embargo en algunas oportunidades no fue posible. Fue muy importante pasar en limpio las notas de campo el mismo día, ya que de esta manera, era más fácil recordar todos los detalles observados en la interacción del aula. Esta labor requirió de una gran cantidad de tiempo, pero fue una parte esencial del proceso investigativo, puesto que al volver a escribirlas, emergían aspectos relevantes que no había percibido en el momento de realizar la visita al salón de clase. Además, las transcripciones diarias me permitieron ir analizando la dinámica del contexto escolar, elaborar preguntas para la educadora, orientar la observación hacia determinados aspectos y elaborar notas 
personales sobre mis sentimientos y mis percepciones en cuanto a lo que ocurría en el salón de clase.

Luego de leer y releer el diario de campo, en el mes de mayo, incluí notas teóricas lo que contribuyó a relacionar la práctica con la teoría. Ese fue un proceso de acción-reflexión que me ayudó a complementar el análisis de la acción pedagógica que se generó en el contexto escolar. El compartir lo que se anotaba en el diario con la educadora, fue una experiencia muy enriquecedora para comprender e interpretar la realidad del aula.

El diario, además, me permitió expresar mis sentimientos, pensamientos, valores y creencias, reflexionar sobre lo que consideraba adecuado y no adecuado para el nivel inicial, creo que me ayudó a crecer como profesional y como persona porque pude ver más allá de lo que esta a la vista, y a la vez, profundizar en los significados que transmitimos en el intercambio social.

\section{Etapa. Compartiendo la información}

Con el propósito de obtener diferentes percepciones sobre la realidad-realidades del aula, dialogaba constantemente con la educadora. Con ella, se comentaban las observaciones. En este intercambio, aclarábamos nuestras percepciones e inquietudes sobre lo que ocurría en el contexto escolar. En este proceso fuimos descubriéndonos como seres humanos y educadoras, lo que nos permitió tomar conciencia de las creencias que fundamentaban nuestra práctica pedagógica e ir realizando cambios en ella, con el propósito de ofrecer una educación más humana y democrática a nuestros estudiantes.

\section{Etapa. Análisis de datos}

El análisis de la información lo realicé de manera progresiva. En primer término, leía los datos y reflexionaba sobre lo vivido para tratar de comprender lo que ocurría en el contexto del aula; transcribía las observaciones y agregaba mi percepción sobre ellas; posteriormente, comentaba las notas con la educadora e incluía sus sugerencias, con el fin de encontrar el significado de la práctica educativa. Traté de leer las situaciones concretas, interpretarlas, visualizarlas de acuerdo con mi experiencia y mi práctica y tomando en cuenta, de una manera muy importante, las reflexiones de la educadora. Este fue un proceso continuo, largo y flexible, de constante elaboración y reelaboración. Para Miguel Martínez, es fundamental recurrir a los sujetos participantes en el proceso investigativo para 
entender, retroalimentar y prevenir falsas interpretaciones de las realidades bajo estudio (1989).

En el mes de mayo tomé el diario de campo, lo leí varias veces para comprender las realidades concretas en sus dimensiones reales y temporales, seguidamente, a partir de los aspectos que sobresalían de las observaciones, las entrevistas y las preguntas de investigación, fui clasificando la información más relevante, asignando un color a los aspectos que sobresalían en las notas: procesos iniciales de lectoescritura, ambiente, tiempo, planeamiento, evaluación, género, relaciones de poder y socialización, por último agregué notas teóricas. De esta manera, elaboré las primeras áreas o temas de análisis en el entendido de que eran flexible, y que constituían una primera aproximación al trabajo con los datos. Al respecto Taylor y Bogdan (1996) afirman:

El análisis de datos es un proceso dinámico y creativo. A lo largo del análisis, se trata de obtener una comprensión más profunda de lo que se ha estudiado, y se continúan refinando las interpretaciones. Los investigadores también se abrevan en su experiencia directa con escenarios, informantes y documentos, para llegar al sentido de los fenómenos partiendo de los datos (1996, p. 159).

Durante los meses de junio, julio, agosto, noviembre y diciembre de 1999 transcribí datos, leí y releí el diario agregué notas personales y traté de develar los significados que subyacen de esa práctica educativa. El análisis fue un proceso profundo, dinámico y sistemático que requirió muchas horas de reflexión y de diálogo para descubrir el significado subjetivo que orientaba las actuaciones de los sujetos en el contexto natural del aula.

En el mes de diciembre incluí notas teóricas al diario y con los datos reelaboré temas o áreas de análisis y construí las siguientes: socialización, procesos de lectoescritura y ambiente de aprendizaje.

Para profundizar en el significado de la acción humana en el salón de clase analicé otras fuentes:

- El planeamiento anual y los planes "quincenales" de febrero a julio; este fue el periodo que observé con mayor intensidad debido a que en agosto una estudiante universitaria empezó su práctica docente en el grupo.

- Trabajos de las niñas y de los niños facilitados por la educadora. 
- El material educativo que había elaborado la profesora para las niñas y los niños.

- Las evaluaciones finales de cada estudiante.

- El relato de experiencia que la educadora había elaborado a principio del año.

- Las notas de las conversaciones informales y de las entrevistas que habíamos realizado en el transcurso del año.

Toda esa información fue consultada una y otra vez en el proceso de análisis. Con base en todos esos datos construí nuevas áreas o temas de análisis después de largas horas de reflexión y análisis de la información. En este proceso fue fundamental el diálogo constante con la educadora que me permitió profundizar en los significados que se transmitieron en el intercambio escolar y que me ayudaron a establecer finalmente las áreas con las que realicé el análisis de la realidad-realidades del aula. Dichas áreas fueron las siguientes:

- La educadora que permitió compartir sus vivencias en el aula

- Construyendo nuestra identidad en el contexto escolar

- Poder, lenguaje y género

- Construyendo el placer de leer y escribir

Estas áreas, a su vez, las dividí en subáreas para realizar el análisis.

Con las áreas definidas, elaboré matrices con cuatro columnas para organizar la información donde incluían las notas del diario, documentos, entrevistas y comentarios de la educadora, mis percepciones y las notas teóricas. Estas matrices se constituyeron en un excelente instrumento para ordenar los datos y realizar el análisis; sin embargo, siempre recurrí a las fuentes originales: mi diario, las entrevistas, los diálogos, los videos, el relato de experiencia, la bibliografía, etc., por lo que estas matrices no fueron estáticas, sino todo lo contrario, ya que muchas veces encontré elementos nuevos para enriquecerlas.

Seguidamente presento un ejemplo de la matriz: 


\begin{tabular}{|c|c|c|c|}
\hline \multicolumn{4}{|c|}{$\begin{array}{l}\text { Área: Procesos iniciales de lectoescritura } \\
\text { Subárea: Los procesos de lenguaje en la definición de temas }\end{array}$} \\
\hline Notas de campo & $\begin{array}{l}\text { Documentos, comentarios, } \\
\text { entrevista con educadora }\end{array}$ & $\begin{array}{l}\text { Percepciones } \\
\text { personales }\end{array}$ & Notas teóricas \\
\hline $\begin{array}{l}\text { E: Ahora, en este } \\
\text { momento, quiero que } \\
\text { me pongan mucha } \\
\text { atención, vamos hacer } \\
\text { algo diferente. En el } \\
\text { kínder hemos } \\
\text { estudiando temas que } \\
\text { yo propongo, como: } \\
\text { las reglas de la clase, } \\
\text { la familia, yo soy yo.... } \\
\text { A partir de ese } \\
\text { momento van a elegir } \\
\text { qué quieren estudiar, } \\
\text { qué quieren aprender. } \\
\text { Van a pensar un } \\
\text { poquito y me van a } \\
\text { decir los temas. Yo los } \\
\text { escribo en este cartel" } \\
\text { (Obs. No15) }\end{array}$ & $\begin{array}{l}\text { "Los dos primeros planes } \\
\text { que realizo durante el año } \\
\text { son quincenales, el primero } \\
\text { se relaciona con la } \\
\text { adaptación y normas; el } \\
\text { segundo se refiere a la } \\
\text { familia. En marzo depende } \\
\text { de la temática propuesta } \\
\text { por los niños, puede durar } \\
\text { una quincena o más, } \\
\text { depende del interés que } \\
\text { manifiesten" (Entrevista } \mathrm{N}^{\circ} \\
1: 15 \text { de febrero). }\end{array}$ & $\begin{array}{l}\text { En un principio, la } \\
\text { educadora tenía } \\
\text { el control absoluto } \\
\text { sobre los temas a } \\
\text { estudiar, pero con } \\
\text { esta actividad se } \\
\text { inicia un cambio. } \\
\text { Es fundamental la } \\
\text { participación de } \\
\text { los niños y las } \\
\text { niñas en la } \\
\text { selección de los } \\
\text { temas, ya que } \\
\text { mediante esta } \\
\text { interacción se } \\
\text { comparte el poder } \\
\text { y se les comunica } \\
\text { indirectamente } \\
\text { que su opinión y } \\
\text { su palabra son } \\
\text { valiosas en el } \\
\text { contexto escolar. }\end{array}$ & $\begin{array}{l}\text { De la lista de temas } \\
\text { seleccionados por los } \\
\text { niños y las niñas, se } \\
\text { identifica el primer } \\
\text { tema de estudio e } \\
\text { ideas de lo que } \\
\text { queremos saber... } \\
\text { (Ruiz,1996, p.94). } \\
\text { Los conocimientos y } \\
\text { las experiencias sirven } \\
\text { de base y forman } \\
\text { parte del currículo } \\
\text { creado en cada salón } \\
\text { (Sáez,1995). }\end{array}$ \\
\hline
\end{tabular}

El análisis no fue fácil ni rápido. Fue un proceso de reflexión continua que en mi caso, se inició desde el primer día que ingresé a la institución, en diciembre de 1998. Desde ese momento, la investigación estuvo latente en todas las acciones de mi vida, en las lecturas, en las conversaciones, en las visitas a otras instituciones educativas, y en mis clases, en fin, en todo lo que hacía.

En algunos momentos me sentí agobiada por la cantidad de información y por todo lo que podía analizar de la vida del aula con los datos que tenía, sin embargo, reflexionando una y otra vez, decidí interpretar lo que me pareció más significativo para comprender la realidad-realidades del salón de clase.

Con base en el análisis, puede develar la acción pedagógica que se generó en un salón de clase de educación inicial y que seguidamente presento como los hallazgos más relevantes del estudio, de acuerdo con la pregunta planteada al inicio de esta investigación. 


\section{HALLAZGOS DE LA INVESTIGACIÓN}

\section{La educadora que me permitió compartir sus vivencias en el aula}

La educadora que participó en esta investigación se desempeñó como una profesional responsable: hizo un cuidadoso diagnóstico de las niñas y los niños, planificaba y organizaba las diferentes estrategias de aprendizaje con ellos y ellas, creaba un ambiente afectivo y de aprendizaje en función de las y los estudiantes, interactuaba con los niños y las niñas atendiendo tanto necesidades grupales como personales; incentivaba el diálogo, la participación y la colaboración; observaba, registraba y valoraba el desarrollo de las estudiantes y los estudiantes, incorporaba a las madres de familia en el trabajo de aula y reflexionaba sobre su labor pedagógica; sin embargo, lo hizo a partir de un interés más práctico que emancipador, puesto que su acción se dirigió a potenciar el desarrollo evolutivo de sus estudiantes y a incentivar la curiosidad infantil .

En sus reflexiones y en su práctica pedagógica, la educadora demostró su preocupación por crear nuevas formas de aprender partiendo de las realidades culturales de los niños y las niñas, pero al no asumirse como una transformadora sociohistórica, reprodujo, en varias ocasiones, el sistema tradicional de enseñanza de la cultura dominante.

Durante la investigación, descubrí que a nivel teórico, muchas veces, creemos que estamos claros en cuanto a ofrecer una educación diferente y emancipadora, no obstante, en la práctica pedagógica, caemos constantemente acciones enajenantes; tal vez por una estructura institucional que no favorece una práctica pedagógica liberadora, o porque no es fácil cambiar visiones de mundo, valores y creencias con las que nos hemos construido como personas desde que nacimos y que se reflejan en nuestro actuar cotidiano.

El ejercer una labor transformadora en el contexto escolar no es un asunto fácil, ni es producto de una investigación; es una cuestión de fondo que lleva tiempo y que requiere de una observación sistemática y crítica de las realidades, del estudio de diferentes autores que permitan ampliar nuestras concepciones; y de una reflexión individual y colectiva de nuestras creencias (concepción de mundo-sociedad-ser humano), es, en definitiva, tomar conciencia de las diferentes realidades para contribuir a transformarlas.

\section{Construyendo identidades en el contexto escolar}

Los procesos de socialización que se dieron en el salón de clase estuvieron fuertemente influenciados por la estructura del sistema educativo, lo que favoreció la 
reproducción de prácticas de la sociedad capitalista-patriarcal. La rigidez en la organización del espacio y del tiempo escolar contribuyeron a la transmisión de símbolos y significados acordes con la ideología dominante.

La institución educativa era pequeña para la cantidad de niños y niñas que albergaba. La decoración no valoraba símbolos de nuestra cultura sino de otras latitudes.

La distribución del tiempo estaba en función de las necesidades institucionales y los grupos eran muy numerosos para ser atendidos por una sola educadora.

El aula estaba recargada de materiales por ser compartida por dos grupos, sin embargo, se respiraba un ambiente cálido y de relativo respeto.

En este contexto, la acción pedagógica favoreció, por una parte, la transmisión de símbolos y de significados acordes con los requerimientos de la cultura dominante, y por otra, promovió prácticas que fomentaron la autonomía, la participación y las relaciones horizontales entre educadora y estudiantes. Fue así como durante los primeros meses del curso lectivo, la educadora asumió una función controladora, pero conforme fue pasando el tiempo, ofreció mayores niveles de autonomía y de acción a los y las estudiantes.

En la selección y la organización de contenidos ella asumió el control al inicio del curso lectivo; trabajó contenidos dirigidos al manejo de la disciplina en el aula y, posteriormente, fue brindando mayor participación a las y los educandos en relación con la selección de los temas a investigar. En este proceso, respetó la decisión de la mayoría. Los niños y las niñas disfrutaron conociendo e investigando sobre plantas, mamíferos, insectos, alimentos y otros temas más.

De la misma forma, al iniciar el ciclo escolar, las actividades tendían a estimular el acatamiento de órdenes y reglas de la institución educativa; sin embargo, luego se observaron transformaciones: poco a poco los niños y las niñas fueron asumiendo roles protagónicos en las diferentes actividades escolares, la educadora promovió la autonomía, el trabajo en equipo y valores como la solidaridad y la cooperación.

Los niños y las niñas "escribieron" cuentos, poesías y cartas, hicieron carteles, murales, maquetas y recetas de cocina, pintaron, dibujaron, escucharon música, y realizaron muchas actividades más. Se les ofrecía oportunidades para conversar, escuchar, "escribir" y "leer", lo que les producía gozo y placer.

No obstante, las transformaciones en el salón de clase no se dieron de una manera lineal ni continua. En algunos momentos se volvía a prácticas controladoras que favorecían la sumisión de las estudiantes y los estudiantes y donde la educadora ejercía el poder verticalmente; en otros momentos, se realizaron acciones que promovían la participación Volumen 2, Número 2, Año 200214 
protagónica de las personas involucradas en el proceso educativo, donde se fomentaba el intercambio de opiniones y las relaciones horizontales entre educadora y estudiantes. Esta situación se dio por dos razones fundamentales: por una parte, la estructura del sistema educativo es rígida e inflexible; por otra, no es fácil para ningún ser humano cambiar una concepción de mundo y una práctica pedagógica que ha venido construyendo a través de la vida. Ella, mediante los procesos de reflexión y análisis de su práctica, se acercaba en ciertas ocasiones, a proveer una acción educativa crítica, pero en otros momentos, reproducía las prácticas de nuestra sociedad capitalista - patriarcal.

\section{Lenguaje, género y relaciones de poder}

En las interacciones del aula, la mayoría de las veces, la docente utilizó un lenguaje sexista en el cual invisibilizaba lo femenino, constituyéndose de esta forma en instrumento de la cultura hegemónica que fortalece las diferencias entre géneros; sin embargo, esta actitud cambió, y en el mes de agosto, empezó a utilizar ambos géneros en su lenguaje oral y en algunos de los materiales que elaboraba para los niños y las niñas. Esta transformación, aunque no fue radical, se dio por tres razones fundamentales:

- Las conversaciones informales entre la educadora y yo, en la que comentábamos lo que sucedía en el aula y buscábamos explicaciones a algunos hechos.

- $\quad$ Los intercambiamos de literatura que posteriormente discutíamos.

- La educadora reflexionaba constantemente sobre su práctica pedagógica con el fin de mejorarla.

El cambio no se dio de manera profunda debido a que se siguieron utilizando otros materiales educativos como cuentos, poesías, canciones y rimas que evidentemente transmiten estereotipos de género que contribuyen a construir identidades y subjetividades de acuerdo con los intereses de una sociedad capitalista y patriarcal.

Fue interesante, además, observar cómo la cultura dominante moldea las actitudes, el pensamiento y el comportamiento de los niños y las niñas mediante sus juegos en las diferentes áreas. Ellos y ellas representaban muy bien las conductas sexistas que la sociedad les asigna según su género, de tal forma las niñas cocinaban, barrían y cuidaban bebés, mientras los niños arreglaban el techo, construían y eran médicos.

En síntesis, los estereotipos de género estuvieron presentes en el contexto escolar de muchas formas: en el lenguaje, en los juegos, en los papeles que asumían las niñas y los niños, en sus gustos, en sus dibujos, y de manera muy clara, en la literatura que se utilizaba 
en el centro infantil, lo que evidenció la influencia que tiene la cultura dominante en el sistema educativo. Por lo tanto, es preciso tomar conciencia de ello para construir una nueva cultura de género que incentive relaciones equitativas entre hombres y mujeres (Lagarde, Marcela,1999). Es claro que este proceso de transformación requiere de tiempo para leer y estudiar teoría crítica, reflexionar y discutir sobre nuestras prácticas cotidianas, de una manera sistemática, pues somos seres humanos que nos hemos construido en un contexto sociohistórico que nos ha introyectado los valores de la cultura capitalista patriarcal, que es preciso transformar.

\section{Construyendo el placer de leer y escribir}

La educadora fue congruente con los principios de la filosofía del Lenguaje Integral, de forma que p00romovió el uso de los cuatro procesos del lenguaje en actividades contextualizadas y significativas, donde los educandos usaron el lenguaje para sus propósitos, para decir o entender algo, es decir, para comunicarse con los demás. Para Kenneth Goodman (1970) el niño y la niña aprenden a leer y a escribir de la misma forma en que aprenden a hablar, ya que aprender el lenguaje es aprender a dar significado y a darle sentido al mundo. Así fue como en el salón de clase, el lenguaje se utilizó de manera natural, se invitó a los niños y a las niñas a conversar de lo que les interesaba, a plantear preguntas, a dar respuestas, a escribir textos, a reflexionar sobre lo escrito, a leer todo tipo de información, a disfrutar de los cuentos, de las poesías y de las canciones, puesto que se "aprende a leer leyendo y a escribir escribiendo".

La educadora partió de los conocimientos que traían los niños y las niñas para ayudarlos a construir nuevos conocimientos, respetó su forma de hablar y los incentivó a participar en las diferentes actividades escolares, compartiendo, de esta manera , el poder con los educandos.

En esa interacción, los niños y las niñas fueron aprendiendo el lenguaje mediante diversas actividades que planeaba la educadora y que los hacía pensar, cuestionar y buscar soluciones a los conflictos. Al respecto, Adelina Arellano afirma:

Los maestros cuya práctica pedagógica parte de la filosofía del Lenguaje Integral son capaces de desafiar a sus alumnos desde muy temprana edad; desde el kínder por medio de lecturas y escritos para que fortalezcan su derecho a decidir, hacer preguntas, a correr riesgos y a desocultar verdades (1995, p.19). 
Mediante el estudio de este caso, encontramos formas diferentes de abordar los procesos de apropiación de la lengua escrita, lo que implicó un cambio epistemológico en la práctica educativa, pues se concibió al sujeto y al objeto como entes activos constructores de conocimiento y no como receptores pasivos de los estímulos del medio. Este cambio no se dio totalmente, pues en el salón de clase se realizaron prácticas de aprestamiento muy arraigadas en nuestros centros infantiles, las cuales representan acciones enajenantes, repetitivas y sin sentido.

A partir de estos hallazgos, es preciso cuestionar las prácticas de aprestamiento, divididas en diferentes áreas, que realizan los niños y las niñas en los centros de educación inicial y que, en muchos casos, representan actividades sin significado, donde se supone que el sujeto aprende por simple repetición, sin tener en cuenta "que ninguna percepción puede darse sin significaciones y esquemas propios del sujeto" (Rojas, Marisela, 1998, p. 27).

\section{REFLEXIONES FINALES}

Los hallazgos de este estudio indican que las transformaciones que deseamos generar en el salón de clase deben partir de procesos de reflexión y de análisis individuales y colectivos. Estos procesos de reflexión requieren de tiempo, puesto que de un día para otro, no es posible desprenderse de lo que históricamente hemos construido en el intercambio social.

Necesitamos tiempo para analizar nuestra visión de mundo, para develar las limitaciones estructurales del sistema educativo, para observar críticamente la realidadrealidades del aula, para develar los símbolos y significados que transmitimos en nuestras interacciones con los demás, para discutir y analizar con otras personas nuestras creencias, valores y acciones, para analizar el papel que desempeñamos en la labor educativa y necesitamos tiempo para estudiar diferentes autores que nos permitan ampliar nuestras concepciones teóricas.

En relación con los procesos iniciales de lecto-escritura, es importante tener claro que la apropiación de la lengua escrita por parte de los niños y las niñas es un proceso histórico y sociocultural que parte de distintas realidades y que se construye y reconstruye en el intercambio con los demás, como lo indican los principios de la Filosofía del Lenguaje Integral. Es necesario tener en cuenta que si valoramos la palabra de nuestros niños y niñas, 
si compartimos el poder con ellos y ellas, si respetamos su forma de hablar, su cultura y sus vivencias vamos a favorecer de manera significativa el aprendizaje de la lengua escrita.

Es preciso, por lo tanto, tomar conciencia que nuestra práctica pedagógica es una acción política-ideológica que va a contribuir a reproducir las desigualdades de la sociedad, o por el contrario, va a ayudar a construir identidades críticas capaces de iniciar procesos de transformación. En la medida que estemos concientes de ello, en esa medida asumiremos una posición ética y política a favor de la justicia, la solidaridad, la paz y la democracia.

En este contexto, es importante preguntarnos ¿Qué tipo de relaciones sociales propiciamos en nuestras aulas? ¿Transferimos el poder a nuestros y nuestras estudiantes? ¿Incentivamos en las y los estudiantes la reconstrucción crítica de acciones y pensamientos? ¿Organizamos actividades que induzcan a la solidaridad, la colaboración y la experiencia compartida? ¿Cómo incentivamos los procesos iniciales de lecto-escritura? ¿Propiciamos el diálogo y la participación auténtica?. Es preciso que nuestra acción pedagógica se base en principios democráticos, como los concebía Dewey, es decir, "más como una forma de vida y una idea moral, que como una mera forma de gobierno, donde los individuos, respetando sus diferentes puntos de vista y proyectos vitales, se esfuerzan a través del debate y la acción política, de la participación y cooperación activa, por crear y construir un clima de entendimiento y solidaridad"(s.r). 


\section{REFERENCIAS}

Alba, Alicia de (1994) Posmodernidad y educación. México D.F., México: Centros de Estudios sobre la Universidad (CESU). 303 p.

Alfaro, Gilberto, Gallard, Alejandro, Tobin, Kenneth. (1991) Creencias, metáforas y metonimias que limitan las posibilidades de cambio en los maestros. Ponencia presentada en el Seminario sobre Investigación Cualitativa en la Educación Latinoamericana. San José, Costa Rica, 15p.

Alfaro, Gilberto. (1994). El educador como aprendiz. Revista de Educación. Universidad de Costa Rica, 18 (1).

Apple, Michael. (1995) La política del saber oficial: ¿Tiene sentido un curriculum nacional? En Volver a pensar la educación. Vol. I. Madrid, España: Morata. pp153-171

Arellano-Osuna, Adelina. (199?). El lenguaje integral : una alternativa para la Educación. Mérida: Venezuela: Venezolana C.A. 133 p. amigo.104 p.

Arellano-Osuna, Adelina. (1997). Lenguaje integral para leer, escribir y aprender. Lima: Libro amigo.104 p.

Bernstein, Basil. (1998) Pedagogía, control simbólico e identidad. Madrid, España: Morata. $239 \mathrm{p}$.

Braslavsky, Berta. (2000) "Las nuevas perspectivas de la alfabetización temprana". En Revista Lectura y Vida. Revista Latinoamericana de Lectura. Buenos Aires, Argentina. Año XX, N 4. Diciembre.2000 pp.32-43.

Chavarría, M. Celina. (1993) Tendencias políticas de la educación preescolar. El dilema de la madre trabajadora. San José, Costa Rica: Editorial de la Universidad de Costa Rica. p.139

Colás, María Paz y Buendía, Lourdes. (1994) Investigación educativa. (2 Ed.) Sevilla, España: Alfar, S.A.

Díaz, María Isabel. (1999) "Algunas reflexiones acerca de: la dimensión de género en el curriculum de la educación parvularia " En Temas Pedagógicos. Serie de Cuadernillos de Estudio. Santiago, Chile: Junta Nacional de Jardines. 18 p.

Facio, Alda. (1994) "Cuando el género suena cambios trae". En Introducción a los estudios de género: categorías básicas de análisis. Managua, Nicaragua: Programa Interdisciplinario de Estudios de Género.

Ferreiro, Emilia. (1983) Psicogénesis de la escritura. En Psicología genética y aprendizajes escolares. Compilación de César Coll. Madrid, España: Siglo XXI pp 79-90..

Freire, Paulo. (1997) Pedagogía del oprimido. 49a edición. México: Siglo Veintiuno.245 p. 
Freire, Paulo. (1998) Pedagogía de la esperanza. $3^{a}$ edición. México: Siglo Veintiuno.

Gadotti, Moacir. (2001). Los aportes de Paulo Freire a la pedagogía crítica. Ponencia en Simposio Latinoamericano de Pedagogía Universitaria "Hacia una pedagogía alternativa para la Educación Superior". 17 al 20 de abril. San José, Costa Rica:Escuela de Formación Docente. Universidad de Costa Rica.

Gimeno, José y Pérez, Ángel. (1993) Comprender y transformar la enseñanza. 2 $2^{a}$ Edición. Madrid: Morata. S.A. 441p.

Giroux, Henry. (1997) .Los profesores como intelectuales. $1^{\text {a }}$ Reimpresión Barcelona, España: PAIDÒS. 279 p.

Giroux, Henry. (1997a) Cruzando límites. Trabajadores culturales y políticas educativas. Barcelona, España: Paidós. 287 p.

Gómez, Margarita. (1994) "La adquisición de la lengua escrita" En Los procesos de lectura y escritura. Selección de lecturas/Com. Por Marta Eugenia Sánchez y Luz Emilia Flores. Heredia: EUNA.pp 55-60

Goodman, Kenneth. (1970) Theoretical models and process of reading. N.J., USA: International Reading Associations

Goodman, Kenneth. (1989). Lenguaje Integral. Mérida,Venezuela: Editorial Venezolana.115p

Goodman, Kenneth y Goodman Yeta. (1993) "Vygotsky desde la perspectiva del lenguaje total" En Vygotsky y la educación. 2a Edición. Buenos Aires, Argentina: Aique pp.263292.

Gore, Jennifer. (1996) Controversias entre las pedagogías. Madrid, España: Morata 199 p

Gurdián, Alicia (1997) Por el derecho a un nuevo imaginario pedagógico. En Revista Reflexiones. Facultad de ciencias Sociales, Universidad de Costa Rica № 65. Diciembre. pp.15-31

Gurdián, Alicia. (1998) "Perfil curricular, temas transversales y derechos humanos" En Reconocer todas las voces de la historia: Derechos Humanos y diversidad cultural en la educación. San José, Costa Rica: CSUCA pp. 65-80.

Gurdián, Alicia. (2001) Tocando la puerta y dejando el recado. ¿Cómo incluir la Equidad de Género en un proyecto curricular en derecho? San José, Costa Rica: ILANUD. Programa Mujer, Justicia y Género. p. 62.

Habermas, Jürgen. (1984) Teoría de la acción comunicativa: complementos y estudios previos. Madrid, España: Cátedra p.507.

Halliday, M. (1973) Explorations in the functions of language. London: Edward Arnold. 
IIMEC. (1999) Paradigma Cualitativo Caracterización. Instituto de Investigación para el Mejoramiento de la Educación Costarricense. Universidad de Costa Rica p.7

Jiménez, Kemly. (1999) Monólogo para un diálogo: acerca de la sociopedagogía. Universidad de Costa Rica. Facultad de Educación. Escuela de Formación Docente. Departamento de Preescolar y Primaria (mimeo).

Lagarde, Marcela. (1999) Una mirada en el umbral del milenio . Instituto de Estudios de la Mujer. UNA. Heredia, Costa Rica. 160 p.

Leal, Aurora y Fortuny, Joan. (1988) Aprendizaje de la lengua escrita en el preescolar y en el ciclo inicial. En Enciclopedia Práctica de Pedagogía . Barcelona: Planeta. Volumen 1, pp. 157-172, 233-264.

Luria, Alexander. (1985) Lenguaje y pensamiento. Segunda Edición. Barcelona, España: Martínez Roca S. A.

Manifiesto por el Derecho de la Esperanza (1997) Universidad de Costa Rica.

Martínez, Miguel. (1989) Comportamiento humano. Nuevos métodos de investigación. México: Trillas $278 \mathrm{p}$.

Matos, José. (1995) El paradigma sociocultural de L. S. Vigostky y su aplicación en la educación. (mimeo).Heredia, Costa Rica: Universidad Nacional

McLaren, Peter. (1997) Pedagogía crítica y cultura depredadora. Barcelona, España: PAIDÒS. 309 p.

Rojas, Marisela. (1998) Educación Científica y Matemática para el niño preescolar I. Perspectiva Constructivista. San José, Costa Rica: EUNED. 171 p.

Rojas, Patricia. (1999) Planeamientos, minutas y crónicas. Nivel de preparatoria. Grupo E. Jardín de Niños Porfirio Brenes Castro. Moravia, Costa Rica $p$

Rojas, Patricia. (1999a) Plan Anual. Nivel de preparatoria. Grupo E. Jardín de Niños Porfirio Brenes Castro. Moravia, Costa Rica 155p

Ruiz, Daisy. (1996) La alfabetización temprana en el ambiente preescolar. San Juan, Puerto Rico: Facultad de Educación, Universidad de Puerto Rico. Recinto Río Piedras. $192 \mathrm{p}$.

Taylor y Bogdan. (1996) Introducción a los métodos cualitativos de investigación. $3^{\text {a }}$ Reimpresión. Barcelona, España; Paidós 343 p.

Woods, Peter. (1995) La escuela por dentro. La etnografía en la investigación educativa. (3 ed.) Madrid: Paidós. 220 p. 